\title{
Freqüência de Candida sp. em biópsias de lesões da mucosa bucal
}

\section{The frequency of Candida sp. in biopsies of oral mucosal lesions}

\author{
Luís Carlos Spolidorio* \\ Vinícius Rangel Geraldo Martins** \\ Ruchele Dias Nogueira** \\ Denise Madalena Palomari Spolidorio*
}

\begin{abstract}
RESUMO: O objetivo desse trabalho foi determinar a freqüência da infecção por Candida sp. em biópsias de lesões da mucosa bucal, assim como associar a presença de Candida sp. com lesões malignas e lesões com vários graus de displasia. Foram utilizadas 832 biópsias da mucosa bucal, previamente incluídas em parafinas, cujos blocos foram obtidos dos arquivos da Disciplina de Patologia da Faculdade de Odontologia de Araraquara da UNESP, no periodo entre 1990-2001. Três cortes seqüenciais foram corados pelo ácido periódico de Schiff (PAS). Do total de biópsias 27,2\% foram PAS positivas, dessas $83,25 \%$ eram provenientes de pacientes do sexo masculino. Houve associação positiva entre infecção com displasia epitelial leve, moderada, severa, carcinoma espinocelular e hiperqueratose $(p<0,05)$. Não houve associação entre hiperplasia fibrosa inflamatória, líquen plano, granuloma piogênico $(p<0,05)$ com infecções fúngicas. A língua foi o sítio mais acometido por infecções em relação a outros sítios ( $<<0,05)$. A partir dos dados quantitativos, concluiu-se que houve correlação positiva de infecção por fungos, lesões displásicas e carcinoma, sendo mais freqüente no sexo masculino. Estes dados não permitem inferir se o fungo causa displasia epitelial e carcinoma, mas confirmam a maior presença de Candida nessas lesões.
\end{abstract}

DESCRITORES: Candida; Câncer oral; Lesões.

\begin{abstract}
Candidosis is the most common fungal infection in the oral cavity, and is usually associated with local and systemic predisposing factors. The ocurrence and relevance of Candidal infection in oral lesions such as liquen planus, leukoplakias and carcinomas are still to be understood. The aim of the present study was to define the frequency of infection by Candida sp. on biopsies of oral mucosal lesions and associate its presence with malignant and dysplastic lesions. Histopathology reports issued between 1990 and 2001 inclusive were reviewed. Three sections of each mucosal biopsy were stained using the periodic acid-Schiff (PAS) technique. From the 832 biopsies $27.2 \%$ were PAS positive, of which $83.25 \%$ were obtained from male patients. There was positive association between fungic infection and mild, moderate and severe epithelial dysplasia, squamous cell carcinoma and hiperqueratosis $(p<0.05)$. There was no association between fungic infection and inflammatory fibrous hyperplasia, hyperkeratosis, lichen planus and pyogenic granuloma $(\mathrm{p}<0.05)$. The frequency of infection in the tongue was significantly higher $(\mathrm{p}<0.05)$ than in the other sites. Our results do not show a causal relation between Candida sp. and dysplastic lesions and carcinomas, but do confirm the higher presence of that microrganism in those lesions.
\end{abstract}

DESCRIPTORS: Candida; Mouth neoplasms; Injuries.

\section{INTRODUÇÃO}

A candidíase ou candidose é a micose mais comum na boca, sendo a Candida albicans a principal espécie encontrada. A infecção por Candida sp. é freqüente no palato de portadores de próteses totais, de glossite rombóide, pacientes imunossuprimidos e que recebem antibioticoterapia ou drogas que causam xerostomia ${ }^{10,13,20}$. A Candida $s p$. pode infectar secundariamente lesões bucais como líquen plano, leucoplasia e carcinoma. O mecanismo de ocorrência, assim como o significado da infecção em cada uma dessas doenças não é estabelecido. Não há evidências da participação da candidiase no desenvolvimento de carcinomas bucais, mas alguns trabalhos mostram que a presença de Candida $s p$. aumenta a severidade das displasias ${ }^{13,20}$. Embora a hiperplasia epitelial, as hiperqueratoses, os microabcessos superficiais e a inflamação crônica da lâmina própria sejam sinais histopatológicos sugestivos da infecção por Candida sp., a hifa hematoxifilica pode ser mais facilmente visualizada quando se faz uso de uma coloração especial, o ácido periódico de Schiff (PAS), que reage com alguns polissacarídeos deste microrganismo, tornando-o positivo em relação às demais estruturas microscópicas ${ }^{7}$.

\footnotetext{
*Professores Assistentes Doutores da Disciplina de Patologia; **Alunos de Iniciação Científica - Faculdade de Odontologia de Araraquara da Universidade Estadual Paulista.
} 
Spolidorio LC, Martins VRG, Nogueira RD, Spolidorio DMP. Freqüência de Candida sp. em biópsias de lesões da mucosa bucal. Pesqui Odontol Bras 2003;17(1):89-93.

Barret et al. ${ }^{2}$ (1998) verificaram que 223 biópsias da mucosa de boca $(4,7 \%$ do total de um período de 5 anos) foram positivas para Candida. Houve associação positiva entre Candida $s p$. com displasia epitelial moderada e severa, glossite rombóide mediana e papilomas escamosos. Apenas 3,1\% dos carcinomas espinocelulares tinham hifas de Candida sp., mas $15,2 \%$ das displasias epiteliais severas e $18 \%$ das moderadas tinham hifas no epitélio. Verificaram também que houve associação negativa entre Candida $s p$. e crescimentos fibrosos benignos, hiperqueratoses, líquen plano e granuloma piogênico. Lipperheid et al. ${ }^{14}$ (1996), sugerem que Candida sp. possui alto potencial de adaptação às alterações associadas com o desenvolvimento de leucoplasias e líquen plano. Roed-Petersen et al. $^{18}$ (1970), encontraram $31 \%$ das leucoplasias positivas para o fungo e $40 \%$ destas mostravam atipia.

A freqüência de candidíase em lesões de mucosa bucal é variável, portanto, o objetivo deste trabalho foi determinar a freqüência da infecção por Candida $s p$. em biópsias de lesões da mucosa bucal coradas pelo PAS, assim como associar a presença de Candida sp. com lesões malignas e lesões com vários graus de displasia.

\section{MATERIAL E MÉTODOS}

Para a realização deste trabalho foram utilizadas 832 biópsias da mucosa bucal, cujos blocos foram obtidos dos arquivos da Disciplina de Patologia da Faculdade de Odontologia de Araraquara da Universidade Estadual Paulista (UNESP), durante o período de 11 de janeiro de 1990 a fevereiro de 2001. As biópsias foram fixadas em formol a $10 \%$ por 24-48 h, lavadas em água corrente por $24 \mathrm{~h}$ e submetidas aos processos rotineiros para inclusão em parafina. Foram obtidos cortes de $5 \mu \mathrm{m}$ de espessura, e estes corados com hematoxilina e eosina (H. E.) para análise microscópica e confirmação do diagnóstico e para tal utilizou-se um microscópio de luz transmitida Zeiss. A classificação dos casos de displasia epitelial foi feita com base no critério proposto por Bánóczy, Csiba ${ }^{3}$ (1976), que utiliza como parâmetros histológicos: estratificação epitelial irregular, hiperplasia da camada basal, processos reticulares em forma de gota, aumento do número de figuras mitóticas, perda de polaridade das células basais, aumento da razão núcleo-citoplasma, polimorfismo nuclear, aumento do tamanho dos nucléolos, ceratinização de células isoladas ou em grupos na camada celular espinhosa e redução da aderência intercelular.
Assim, os casos de displasia podem ser caracterizados como:

- displasia leve: quando duas características citadas anteriormente estão presentes.

- displasia moderada: quando duas a quatro das características citadas estão presentes.

- displasia severa: quando cinco ou mais das características citadas estão presentes.

Com a confirmação do diagnóstico, outros 3 cortes semi-seriados de $3 \mu \mathrm{m}$ de espessura, obtidos dos mesmos blocos de que se retiraram os cortes para análise em H. E., foram colocados em lâminas preparadas com adesivo à base de 3-aminopropiltrietoxisilano (Sigma Chemical CO., St Louis, MO EUA); em seguida submeteram-se a coloração pelo PAS, para identificar o fungo e a sua localização.

Os dados referentes à localização da lesão, idade, sexo foram obtidos dos respectivos prontuários dos pacientes. Foram selecionadas aquelas fichas que não faziam menção a presença de doença sistêmica, que incluíam: alterações hormonais, diabetes, hipotiroidismo, hiperparatiroidismo, supressão adrenal, imunossupressão. Foram excluídos, também, os prontuários dos pacientes que faziam uso de antibióticos ou drogas que causam xerostomia, que são fatores que predispõem a infecções por Candida (Farah et al. ${ }^{8}, 2000$ ).

Os dados obtidos foram submetidos a análise estatística pelo teste $t$ de Student.

\section{RESULTADOS}

Das 832 biópsias utilizadas neste trabalho, 227 $(27,2 \%)$ apresentaram infecção por Candida sp., que se apresentaram caracteristicamente na forma de hifas. Os casos de carcinoma espinocelular, os vários graus de displasia, as hiperqueratoses, os papilomas escamosos apresentaram positividade para o fungo significativamente superior $(\mathrm{p}<0,05)$ quando comparado ao aumento gengival induzido por drogas, líquen plano, granuloma piogênico e à hiperplasia fibrosa inflamatória (Tabela 1).

Das lesões infectadas, o número de biópsias provenientes de pacientes do sexo masculino $(83,25 \%)$ foi significativamente superior $(p<0,05)$ às biópsias provenientes de pacientes do sexo feminino $(16,75 \%)$, como mostra a Tabela 2.

A região mais afetada na cavidade bucal foi a língua, seguida pelo assoalho bucal, mucosa jugal e gengiva. Nenhuma lesão infectada por Candida $s p$. foi encontrada no palato dos pacientes biopsiados, como mostra a Tabela 3. 
Spolidorio LC, Martins VRG, Nogueira RD, Spolidorio DMP. Freqüência de Candida sp. em biópsias de lesões da mucosa bucal. Pesqui Odontol Bras 2003;17(1):89-93.

TABELA 1 - Incidência de infecção por Candida sp. em lesões de mucosa bucal diagnosticadas pelo PAS.

\begin{tabular}{l|c|c}
\hline \hline \multicolumn{1}{c|}{ Lesão } & $\begin{array}{c}\mathrm{N}^{\circ} \text { de lesões } \\
\text { diagnosticadas }\end{array}$ & $\begin{array}{c}\text { Percentagem de } \\
\text { lesões infectadas }\end{array}$ \\
\hline Hiperqueratose & 76 & $42,1^{*}$ \\
\hline Displasia epitelial leve & 103 & $42,7^{*}$ \\
\hline $\begin{array}{l}\text { Displasia epitelial } \\
\text { moderada }\end{array}$ & 101 & $26,7^{*}$ \\
\hline Displasia epitelial severa & 111 & $41,4^{*}$ \\
\hline Carcinoma espinocelular & 109 & $44,9^{*}$ \\
\hline Papiloma escamoso & 96 & $20,8^{*}$ \\
\hline $\begin{array}{l}\text { Aumento gengival } \\
\text { induzido por drogas }\end{array}$ & 38 & 0 \\
\hline Líquen plano & 54 & 16,7 \\
\hline Granuloma piogênico & 54 & 0 \\
\hline $\begin{array}{l}\text { Hiperplasia fibrosa } \\
\text { inflamatória }\end{array}$ & 90 & 227 \\
\hline Total & 832 & 0 \\
\hline \hline
\end{tabular}

*Estatisticamente significante pelo teste $t$ de Student ao nivel de $5 \%$.

O padrão histopatológico da candidíase bucal era variável. As características encontradas em comum, incluíram espessura de ceratina e alongamento das papilas epiteliais. No conjuntivo subjacente encontrava-se variável infiltrado inflamatório mononuclear.

\section{DISCUSSÃO}

A infecção por Candida é freqüente no palato de portadores de próteses totais, na glossite rombóide, imunossupressão e naqueles que recebem antibioticoterapia ou drogas que causam xerostomia. A Candida pode infectar secundariamente lesões bucais como líquen plano, leucoplasia e carcinoma, porém a freqüência do mecanismo de ocorrência, assim como o significado da infecção em cada uma dessas doenças não é conhecido ${ }^{4,15,17}$.

Segundo Roed-Petersen et al. ${ }^{18}$ (1970), quando se emprega o PAS, há $13 \%$ de chance de não se detectar o fungo, particularmente se as hifas forem escassas e se apenas um corte da biópsia for analisado. Assim utilizamos 3 cortes semi-seriados distantes uns dos outros de aproximadamente $9 \mu \mathrm{m}$, na tentativa de excluir esse fator.

O câncer bucal é responsável por cerca de 1\% das mortes por câncer. A etiologia envolve numerosos fatores, sendo os mais importantes o tabaco e o
TABELA 2 - Número de lesões PAS-positivas em relação ao sexo.

\begin{tabular}{l|c|c}
\hline \hline \multicolumn{1}{c|}{ Lesão } & $\begin{array}{c}\mathrm{N}^{\circ} \text { de lesões } \\
\text { infectadas }\end{array}$ & $\begin{array}{c}\text { Percentagem de } \\
\text { indivíduos do } \\
\text { sexo masculino }\end{array}$ \\
\hline Hiperqueratose & 32 & $87,5^{*}$ \\
\hline Displasia epitelial leve & 44 & $77,27^{*}$ \\
\hline $\begin{array}{l}\text { Displasia epitelial } \\
\text { moderada }\end{array}$ & 27 & $85,18^{*}$ \\
\hline Displasia epitelial severa & 46 & $84,78^{*}$ \\
\hline Carcinoma espinocelular & 49 & $91,83^{*}$ \\
\hline Papiloma escamoso & 20 & $55^{*}$ \\
\hline Líquen plano & 9 & $100^{*}$ \\
\hline Total & 227 & 83,25 \\
\hline \hline
\end{tabular}

*Estatisticamente significante pelo teste $t$ de Student ao nivel de $5 \%$.

álcool, e a maioria dos casos de câncer bucal resulta da ação de carcinógenos exógenos, sendo que alguns deles agem sinergicamente. Herpes simples, papilomavirus humano (HPV) e Candida $s p$. já foram associados à etiologia do câncer bucal, embora o papel de microrganismos, nesse câncer ainda seja controverso ${ }^{4,11}$.

Nós pudemos observar uma associação significante entre a infecção fúngica e a displasia epitelial, nos seus variados graus, e o carcinoma escamoso. Tais resultados sugerem associação da infecção fúngica com a presença de displasias epiteliais e carcinoma, como descrito por Cawson, Binnie $^{5}$, (1980); Field et al. ${ }^{9}$ (1989) e Barret et al. $^{2}$ (1998). Esse último grupo de autores também encontrou associação positiva entre papiloma escamoso e infecção por Candida sp..

Valores positivos para associação entre infecções por Candida $s p$. e leucoplasias foram relatados por vários autores como Roed-Petersen et al. ${ }^{18}$ (1970), Rindum et al. ${ }^{17}$ (1994), porém com porcentagens variáveis. Os extensivos trabalhos de Reenstrup $^{16}$ (1970) e Silverman et al. ${ }^{19}$ (1984), que analisaram 235 e 257 biópsias respectivamente, relataram que $23 \%$ e $25 \%$ das lesões continham infecção fúngica. Nesses estudos, o número de cortes examinados não foi relatado, mas Reenstrup ${ }^{16}$ (1970), afirmou que, nos casos PAS positivos, $25 \%$ das lesões apresentaram displasia.

Das lesões apresentadas no presente trabalho, aproximadamente $27,2 \%$ estavam infectadas por hifas de Candida sp., sendo que 14,06\% das lesões infectadas apresentaram displasia epitelial. De 
Spolidorio LC, Martins VRG, Nogueira RD, Spolidorio DMP. Freqüência de Candida sp em biópsias de lesões da mucosa bucal. Pesqui Odontol Bras 2003;17(1):89-93.

TABELA 3 - Distribuição das lesões de mucosa PAS-positivas de acordo com a região anatômica.

\begin{tabular}{l|c|c|c|c|c}
\hline \hline \multicolumn{1}{c|}{ Lesão } & Língua & Assoalho bucal & Palato & Gengiva & Mucosa jugal \\
\hline Hiperqueratose & 15 & 10 & 0 & 7 & 0 \\
\hline Displasia epitelial leve & 16 & 23 & 0 & 0 & 5 \\
\hline Displasia epitelial moderada & 9 & 9 & 0 & 0 & 9 \\
\hline Displasia epitelial severa & 18 & 20 & 0 & 0 & 8 \\
\hline Carcinoma espinocelular & 20 & 25 & 0 & 0 & 4 \\
\hline Papiloma escamoso & 8 & 0 & 0 & 0 & 12 \\
\hline Líquen plano & 0 & 0 & 0 & 0 & 9 \\
\hline \hline
\end{tabular}

acordo com o que foi discutido anteriormente, podemos afirmar que esses valores estão de acordo com a literatura revisada e que há relação positiva entre o fungo e a displasia epitelial. Como citado anteriomente, Candida $s p$. é freqüentemente isolada de lesões malignas e potencialmente malignas na boca, entretanto seu papel na etiologia dessas lesões é incerto. A interpretação mais aceita para a presença de Candida $s p$. nessas lesões é que ela seja um colonizador secundário da mucosa previamente alterada. Histologicamente, as leucoplasias apresentam hiperqueratose, o que pode explicar a constante presença de Candida sp., que é queratolítica. Alguns tipos de Candida sp. estão associados com a etiologia do câncer bucal devido a produção de nitrosaminas endógenas. Em algumas regiões da China é alto o índice de carcinoma de esôfago, provavelmente relacionado com a alta concentração de nitrato e nitrito na água, os quais constituem os precursores do carcinógeno benzilmetilnitrosamina. C. albicans é freqüentemente isolada desses individuos. Hsia et al. ${ }^{12}$ (1981) demostraram que biotipos de Candida sp. têm a capacidade de aumentar a produção de benzilmetilnitrozamina, através de seus precursores, sugerindo um papel importante da Candida sp. no desenvolvimento de carcinoma de esôfago nesses pacientes.

Silverman et al. $^{20}$ (1985), avaliando pacientes portadores de líquen plano, concluíram que a infecção fúngica é um fator insignificante nesta doença, assim como Cawson ${ }^{4}$ (1966), que investigou 27 casos de líquen plano e não encontrou nenhum caso relacionado positivamente ao fungo. Observamos resultados semelhantes quando analisamos os 54 casos de líquen plano presentes nesse estudo.

No presente trabalho, das 227 lesões infectadas, 189 foram obtidas de pacientes do sexo masculino. Isso pode ser explicado por um fator social, já que o índice de homens fumantes, é superior ao indice de mulheres que usam tabaco (Barret et al. ${ }^{2}$,
1998; Daftary et al. $\left.{ }^{6}, 1972\right)$. O papel do tabaco na candidiase bucal é controverso. O aumento da queratinização do epitélio em pacientes fumantes parece facilitar a aderência de Candida ao epitélio. Arendorf et al. ${ }^{1}$ (1980) observaram relação entre infecções na mucosa bucal e tabagismo, entretanto outros estudos não observam essa relação (Barret et al. ${ }^{2}, 1998$; Daftary et al. $\left.{ }^{6}, 1972\right)$. O sítio bucal que apresentou o maior número de lesões infectadas foi a língua $(73,22 \%)$, seguida pela mucosa jugal $(22,95 \%)$ e gengiva $(3,83 \%)$. Nenhuma lesão infectada foi encontrada no palato. De acordo com Arendorf, Walker ${ }^{1}$ (1980), a lingua e a mucosa labial foram os lugares mais vulneráveis à infecção. Outros sítios vulneráveis são aqueles compostos por um epitélio estratificado pavimentoso paraqueratinizado ou não queratinizado, que formam uma barreira física de proteção mais fraca para a penetração dos microrganismos do que a de um epitélio ortoqueratinizado.

$\mathrm{Na}$ confirmação da infecção fúngica de lesões displásicas, papiloma escamoso e carcinoma a terapia antifúngica dever ser considerada (Barret et $\left.a .^{2}, 1998\right)$.

\section{CONCLUSÕES}

Houve correlação positiva entre infecção por Candida sp. e displasia epitelial nos seus variados graus, papiloma escamoso e carcinoma espinocelular, quando comparado ao aumento gengival induzido por drogas, líquen plano, granuloma piogênico e hiperplasia fibrosa inflamatória.

A coloração pelo PAS deve ser usada nas ocasiões onde a displasia epitelial for diagnosticada pelas colorações de rotina.

Os dados do presente trabalho não permitem inferir se a Candida causa displasia epitelial e carcinoma, mas confirmam a maior presença de Candida nessas lesões. 
Spolidorio LC, Martins VRG, Nogueira RD, Spolidorio DMP. Freqüência de Candida sp. em biópsias de lesões da mucosa bucal. Pesqui Odontol Bras 2003;17(1):89-93.

\section{REFERÊNCIAS}

1. Arendorf TM, Walker DM. The prevalence and intra-oral distribution of Candida albicans in man. Arch Oral Biol 1980;25:1-10.

2. Barret AW, King-Smill VJ, Speight PM. The frequency of fungal infection in biopsies of oral mucosal lesions. Oral Dis 1998;4:26-31.

3. Bánóczy JC, Csiba A. Occurrence of epithelial dysplasia in oral leukoplakia. Analysis and follow-up study of 12 cases. Oral Surg Oral Med Oral Pathol 1976;42:766-74.

4. Cawson RA. Cronical oral candidiasis and leukoplakia. Oral Surg Oral Med Oral Pathol 1966;22:582-91.

5. Cawson RA, Binnie WH. Candida, leukoplakia and carcinoma: a possible relationship. In: Mackenzie, IC; Dabelsteen, E; Squier, CA. Oral Premalignancy. Ames: University of Iowa Press; 1980. p.59-66.

6. Daftary DK, Mehta FS, Gupta PC, Pindborg JJ. The presence of Candida in 723 oral leukoplakias among Indian villagers. Scand J Dent Res 1972;80:75-9.

7. Epstein JB, Komiyama K, Duncan D. Oral tropical steroids and secondary oral candidiasis. $J$ Oral Med 1986;41:223-7.

8. Farah CS, Ashaman RB, Challacombe SJ. Oral Candidosis. Clin Dermatol 2000;18:553-62.

9. Field A, Field JK, Martin MV. Does Candida have a role in oral epithelial neoplasia? J Vet Med Mycol 1989;27:277-94.

10. Fotos PG, Hellstein JW. Candida and candidosis. Epidemiology, diagnosis and therapeutic management. Dent Clin North Am 1992;36:857-78.

11. Gerson SJ. Oral cancer. Crit Rev Oral Biol Med 1990;1:153-66.
12. Hsia CC, Sun TT, Wang YY, Anderson L, Armstrong D, Good RA. Enhancement of formation of the esophageal carcinogen benzylmetylnitrosamine from its precursors by Candida albicans. Proc Natl Acad Sci 1981;78:1878-81.

13. Lilienthal B. Studies of flora of the mouth - III. Yeast-like organisms: some observations on their incidence in the mouth. Aus J Exp Biol Med Sci 1950;28:279-86.

14. Lipperheid V, Quindos G, Jimenes Y, Ponton J, Bagan-Sebastian JV, Aguirre JM. Candida biotypes in patients with oral leukoplakia and lichen planus. Mycophathologia 1996;134:75-82.

15. McCullough MJ, Ross BC, Reade PC. Candida albicans: a review of its history, taxonomy, epidemiology, virulence attributes, and methods of strain differentiation. Int $\mathrm{J}$ Oral Maxillofac Surg 1996;25:136-44.

16. Reenstrup G. Occurrence of Candida in oral leukoplakias. Acta Pathol Microbiol Scand [B] Microbiol Immunol 1970;78:421-4.

17. Rindum JL, Stenderup A, Holmstrup P. Identification of Candida albicans types related to healthy and pathological oral mucosa. J Oral Pathol Med 1994;23:323-8.

18. Roed-Petersen B, Renstrup G, Pindborg JJ. Candida in oral leukoplakias. A histologic and esfoliative cytologic study. Scand J Dent Res 1970;78:323-8.

19. Silverman S, Gorsky M, Lozada-Nur F. Oral Leukoplakia and malignant transformation. A follow-up study of 257 patients. Cancer 1984;53:563-8.

20. Silverman S, Gorsky M, Lozada-Nur F. A prospective follow-up study of 570 patients with oral lichen planus: persistence, remission and malignant association. Oral Surg Oral Med Oral Pathol 1985;60:30-4.
Recebido para publicação em 30/10/01

Enviado para reformulação em 20/06/02 Aceito para publicação em 18/10/02 